\title{
Handling Conjunctions in Named Entities
}

\author{
Robert Dale $^{1}$ and Paweł Mazur ${ }^{1,2}$ \\ ${ }^{1}$ Centre for Language Technology, Macquarie University, \\ NSW 2109, Sydney, Australia \\ \{rdale, mpawel\}@ics.mq.edu.au \\ ${ }^{2}$ Institute of Applied Informatics, Wrocław University of Technology \\ Wyb. Wyspiańskiego 27, 50-370 Wrocław, Poland \\ Pawel.Mazur@pwr.wroc.pl
}

\begin{abstract}
Although the literature contains reports of very high accuracy figures for the recognition of named entities in text, there are still some named entity phenomena that remain problematic for existing text processing systems. One of these is the ambiguity of conjunctions in candidate named entity strings, an all-too-prevalent problem in corporate and legal documents. In this paper, we distinguish four uses of the conjunction in these strings, and explore the use of a supervised machine learning approach to conjunction disambiguation trained on a very limited set of 'name internal' features that avoids the need for expensive lexical or semantic resources. We achieve $84 \%$ correctly classified examples using $k$-fold evaluation on a data set of 600 instances. Further improvements are likely to require the use of wider domain knowledge and name external features.
\end{abstract}

\section{Introduction}

Named entity recognition consists of identifying strings in a text that correspond to named entities, and then classifying each such named entity string as being of a specific type, with typical categories being Company, Person and Location. The range of named entity categories to be identified is usually application dependent.

Introduced for the first time as a separately evaluated task at the Sixth Message Understanding Conference in 1995 (see, for example [112]), named entity recognition has attracted a considerable amount of research effort. Initially handled with hand crafted rules (as, for example, in many of the participating systems in MUC-6 and MUC-7) and later by means of statistical approaches (see [3[4]), the state-of-the-art provides high performance for named entity identification and classification both for specific domains and for language- and domain-independent systems.

However, our experience with existing software tells us that there are still some categories of named entities that remain problematic. In particular, relatively little work has explored the disambiguation of conjunctions appearing in named entity strings. Resources such as an appropriate domain lexicon or relevant semantic knowledge might allow a system to emulate a human's ability to determine that a string like Seshasayee Paper and Boards Limited is a single company name; but in the absence of such resources, the string could just as easily be interpreted as two separate names. Determining the correct interpretation is clearly important for any application which relies

A. Gelbukh (Ed.): CICLing 2007, LNCS 4394, pp. 131-142 2007.

(C) Springer-Verlag Berlin Heidelberg 2007 
on named entity extraction. We are interested in how such interpretations can be arrived at relatively cheaply, and particularly without recourse to expensive-to-construct resources, so as to allow for rapid development in new domains.

The significance of this kind of ambiguity depends, of course, on the extent to which the phenomenon of conjunctions in named entities is widespread. Our current work focuses on a corpus of 13000 company announcements released through the Australian Stock Exchange: these are documents provided by companies in order to meet both continuous and periodic disclosure requirements, in which we want to track mentions of companies and individuals across time.

From this corpus, we selected 45 documents at random; in these documents, there were a total of 545 candidate named entity strings, of which 31 contained conjunctions. This informal sampling suggests that conjunctions appear, on average, in around $5.7 \%$ of candidate named entity strings; however, in some documents in our sample, the frequency is as high as $23 \%$. For comparison, in the MUC-7 evaluation data, the proportion of candidate named entity strings containing conjunctions is $4.5 \%$. The documents in our corpus have some features that are not necessarily typical for other corpora. In particular, texts in this domain frequently have some of the characteristics of legal documents, where many sometimes apparently arbitrary elements are given initial capitals. Therefore, we might expect some specific domains, such as those dealing with accountancy and law, to have a higher density of names involving conjunctions. These frequencies are sufficient to suggest that the seeking of an appropriate means of handling conjunctions is a worthwhile and important pursuit.

The paper is structured as follows. In Section 2, we provide a characterisation of the problem to be addressed, and in Section 3 we summarise some related work. In Section 4 we describe the data used in our experiments, the name-internal text features used as attributes for classification, and the data encoding used to encode the features into a feature vector. Then, in Section 5, we discuss how we determined a baseline for our experiments, and describe the machine learning algorithms we used. Section 6 provides a discussion of the evaluation scheme we adopted, and an overview of the results achieved in the experiments. Section 7 presents details of what went wrong by analysing misclassified examples from our data set. Finally, in Section 8 , we present a discussion of possible directions in which the approach described here could be further developed.

\section{Problem Description}

An examination of the candidate named entity strings appearing in our corpus reveals four distinct uses of the conjunction, as exemplified in the following examples:

1. Oil and Gas Ltd

2. Agfa and Fuji

3. John and Mary Smith

4. Company Secretary Resignation and Appointment

In example (1), we have a single named entity that happens to contain an internal conjunction; in example (2), we have a conjunction of two distinct named entities; and in 
examples (3) and (4), we have conjunctions that, from a linguistic perspective, contain a form of ellipsis, so that one conjunct is incomplete on its own, but can be completed using information provided in the other conjunct. Correspondingly, we distinguish four categories of candidate named entity strings containing conjunctions.

Name Internal Conjunction (NI): This category covers those cases where the candidate named entity string contains one named entity, where the conjunction is part of the name. Some examples from our corpus: Publishing and Broadcasting Limited, $J$ B Were \& Son, Hancock and Gore, and Acceptance and Transfer Form.

Name External Conjunction (NE): This category covers those cases where the conjunction serves to separate two distinct named entities. Some examples from our corpus: Italy and Central Europe, Hardware \& Operating Systems, Mr Danny Fisher and Mr Don Wilson, and American Express and Visa International.

Right-Copy Separator (RC): This category of conjunction separates two named entities, where the first is incomplete in itself but can be completed by copying information from the right-hand conjunct. This is perhaps most common in conjunctions of proper names, as in John and Mary Smith, but appears in other contexts as well. Some examples from our corpus: State and Federal Government, Eastern and Western Australia, and General \& Miscellaneous Equipment.

Left-Copy Separator (LC): This is similar to the above category, but instead of copying information from the right-hand conjunct, to complete the constituent named entities we need to copy information from the left conjunct. Examples in our corpus: Gas Supply and Demand, Financial Statements and Reports, Hospital Equipment \& Systems, J H Blair Company Secretary \& Corporate Counsel.

Conceptually, we might view the last two categories as subtypes of the more general category Copying Separator; however, we keep the two categories separate since the process of reconstructing the unelided conjuncts is different in each case.

Our approach to the problem of determining the type of a conjunction in a candidate named entity string is to use a machine-learned classifier. We are particularly interested in seeing how far we can address the task using only limited knowledge sources: in the work described here, we restrict ourselves to very limited gazetteers that contain the most frequent proper nouns that appear in our corpus, and to the use of so-called 'nameinternal' properties (i.e., characteristics of the candidate string itself, rather than of its surrounding context). Using only limited gazetteers maximises portability; considering only name internal properties will make it easier to see the impact of subsequently adding contextual information. Perhaps more importantly with regard to the specific data we are dealing with, we find many candidate strings appearing in typographic contexts such as tables where the relevant local context can be hard to determine, if it exists at all; in such cases, all we can rely on are the name-internal features.

\section{Related Work}

One of the first approaches to named entities containing conjunctions is reported in [5]. This work dealt with only two categories of conjunctions (those we have termed Name Internal and Name-External), but also considered the use of commas as conjunctions. 
Their solution was based on heuristics using the syntactic number of the verb used with the candidate named entity string and the number of conjuncts in the expression (a large number suggests a Name External conjunction). The reference to name-external syntactic information here means that this approach would not work for much of our data, which is contained in tables.

Coates-Stephens [6] describes the FUNES system, developed for the acquisition of proper names and their descriptions from free text. This work covers all four categories of conjunction that we have identified; the solution is based on the identification of the syntactic number or keywords in a candidate named entity string or in its description, for example in apposition.

McDonald's Sparser [7] uses hand-written rules that make use of name-internal and name-external features of candidate named entity strings; The approach produces nearly $100 \%$ correct results on a selected sublanguage for "Who's News" articles from the Wall Street Journal, but McDonald notes that a new implementation would be required in order to apply the approach to a more diverse set of texts.

Mikheev et al. [8] suggested the strategy of examining the preceding document context to identify candidate conjuncts that should be considered as separate named entities. Mikheev et al. mention this approach being part of their system used in the MUC-7 competition, but no data is reported on the accuracy of this kind of heuristic; in our experience, there are many cases where there are no antecedent mentions that can be used in this way. Furthermore, in the MUC-7 data Left- and Right-Copy categories are not distinguished from Name-Internal.

In more recent work of relevance, we would point to the novel approach to segmentation described in [9]. Using multilabel classification, it is possible to tag overlapping and non-contiguous segments. However, to our knowledge there are no available results to indicate how well this approach would work for the conjunction disambiguation problem. Other work [10] has used the presence of a conjunction as a feature in machine-learning-based NER, but it is unclear what benefits were gained by introducing this feature.

More generally, of course, the processing of conjunctions has been a focus of interest in linguistics; in particular, Categorial Grammar (see, for example, [11]) provides a sophisticated treatment of the syntax of conjunctions.

\section{Experimental Setup}

\subsection{Corpus and Data Preparation}

The focus of our project is a data set from the Australian Stock Exchange (ASX). This data set consists of a large number of company announcements: for a variety of regulatory reasons, listed companies provide around 100000 documents to the ASX each year, and the ASX subsequently makes these available to users via the web. For more information about the documents in this corpus, and a discussion of our general approach to processing them, see [12].

The corpus used for our research consisted of a 13460 document sub-corpus drawn from a larger corpus of company announcements from the ASX. The documents range in length from 8 to 1000 lines of text. 
Table 1. Example distributions in categories

\begin{tabular}{|c|c|c|c|c|}
\hline NI & NE & RC & LC & Sum \\
\hline \hline 185 & 350 & 39 & 26 & 600 \\
$30.8 \%$ & $58.3 \%$ & $6.5 \%$ & $4.3 \%$ & $100 \%$ \\
\hline
\end{tabular}

Evaluation data was prepared as follows. For our purposes, we define a candidate named entity string to be any sequence of words with initial capitals and one embedded conjunction. We also allowed these strings to contain the lowercased preposition of and the determiners a, an, and the. Candidate named entity strings from sentences written completely in uppercase or with every word being initcapped (i.e., strings in 'title case') were ignored. Using a Perl script, we extracted 10925 candidate named entity string instances from our corpus, corresponding to 6437 unique forms. From the set of unique forms, we randomly selected 600 examples for our test data set. In a small number of cases, problems arising from typographic features such as ASCII formatted tables caused us to manually correct some individual strings. An example of the need for such correction is demonstrated by the candidate extracted string Name of Entity Hancock \& Gore Limited, where it turns out that Name of Entity is a label in a list, and Hancock \& Gore Limited, being a company name, is the value of that label; however, in our data, the text extraction process has caused the separating formatting to be lost, resulting in the two strings being concatenated. In this case we remove Name of Entity from the string extracted by our Perl script, on the assumption that a smarter text extraction technique would be able to interpret the layout more accurately.

The resulting set of strings was then annotated using a set of small gazetteers listing common person names, company names, locations and other elements that are frequent in our corpus and related to our tagset, which is described in the next section 1

The categories of the conjunctions in the candidate named entity strings were assigned by a human annotator. Table 1 presents the distribution of evaluation instances across the four conjunction categories introduced above.

\subsection{The Tag Set}

We developed a 16-tag tag set, presented in Table 2, to annotate the tokens in our corpus of candidate named entity strings. Most of the tags, such as Loc, Org, GivenName, AlphaNum, Dir, and PersDesig, are the same as those used by many other named entity recognizers; some, however, are specific to our needs. The Son tag is used to annotate tokens whose surface form is either Son or Sons: these occur relatively often in company names (as, for example, in A Davies \& Sons Pty Ltd), and are a strong indicator of the Name Internal Conjunction category. The Of and Det tags are used to mark the preposition of and the determiners the, a and an, irrespective of casing. Finally, InitCapped is used to annotate any tokens that do not belong to the other categories, or which are ambiguous between those categories.

\footnotetext{
${ }^{1}$ This is part of our strategy for fast deployment in a new domain, where a seed lexicon is
} constructed from the most frequent words that contain initial capitals. 
Table 2. The tagset used for text annotation

\begin{tabular}{|r|l|l||l|l|l|}
\hline No & Tag & Meaning & No & Tag & Meaning \\
\hline \hline 1 & Loc & The name of a location & 9 & CompDesig & A company designator \\
2 & Org & The name of an organization & 10 & Son & Son $(s)$ \\
3 & GivenName & A person's given name & 11 & Dir & A compass direction \\
4 & FamilyName & A person's family name & 12 & AlphaNum & An alphanumeric expression \\
5 & Initial & An initial in the range A-Z & 13 & Month & The name of a month \\
6 & CompPos & A position within a company & 14 & Of & Preposition of \\
7 & Abbrev & Abbreviation & 15 & Det & Determiners the, a, an \\
8 & PersDesig & A person designator & 16 & InitCapped & Unrecognized initcapped token \\
\hline
\end{tabular}

Table 3. The popularity of tags in annotated data

\begin{tabular}{|l|r|r|}
\hline \multicolumn{1}{|c|}{ Tag } & Occurrences & Percentage \\
\hline \hline InitCapped & 925 & 42.24 \\
Loc & 245 & 11.19 \\
Org & 175 & 7.99 \\
FamilyName & 164 & 7.49 \\
CompDesig & 138 & 6.30 \\
Initial & 108 & 4.93 \\
CompPos & 99 & 4.52 \\
GivenName & 89 & 4.06 \\
\hline
\end{tabular}

\begin{tabular}{|l|r|r|}
\hline \multicolumn{1}{|c|}{ Tag } & Occurrences & Percentage \\
\hline \hline Of & 76 & 3.47 \\
Abbrev & 73 & 3.33 \\
PersDesig & 39 & 1.78 \\
Det & 31 & 1.42 \\
Dir & 12 & 0.55 \\
Son & 7 & 0.32 \\
Month & 6 & 0.27 \\
AlphaNum & 3 & 0.14 \\
\hline
\end{tabular}

We also recognize multi-word elements where there is no ambiguity (for example, in the case of unambiguous person, location and company names). For example, although the company name Australia and New Zealand Banking Group Limited is not in our gazetteer, New Zealand as a country name is, and so this string is recognized as a sequence of tokens whose types are marked as Loc and Loc Org CompDesig; here the second Loc tag corresponds to the pair of tokens New Zealand.

We refer to the sequence of tags assigned to a particular string as a pattern. A pattern also indicates the conjunction type present in the string, as determined through the human annotation; so, for the example above, the complete pattern is 〈Loc and Loc Org CompDesig, Internal〉.

Table 3 presents the number of tags of each type used to annotate our data set; in total there were 2190 tags assigned over the 600 candidate named entity strings, for an average of 3.65 tags per instance.

Notably, a significant number of the tokens are tagged as simply being of type InitCapped; this is in keeping with our deliberate use of small gazetteers, and is likely to be the case in any domain where new names are constantly being introduced.

\subsection{Encoding}

For the purposes of machine learning, we encode each pattern in the following way. We create an attribute for each of the 16 tag types for each of the left and right sides of a 
conjunction, for a total of 32 attributes. The attributes are of integer type with values $\{0,1\}$, thus signaling either the presence or absence of a token of that type anywhere within either conjunct. We also introduce an addition binary attribute, ConjForm, for encoding the lexical form of a conjunction in the string: 0 denotes $\& ; 1$ denotes and.

With each data instance there is associated a categorical ConjType attribute with the values $\{$ Internal, External, Right-Copy, Left-Copy $\}$; this is used to encode the actual category of the conjunction in the string.

\section{The Algorithms}

\subsection{Baseline}

It is quite common to determine a baseline using the 0-R algorithm, which simple predicts the majority class [13]. On our data set, with this approach we get a baseline accuracy of $58.33 \%$. However, we have found that with the 1-R algorithm, described in [14], we obtain a better-performing model based simply on the lexical form of the conjunction:

IF ConjForm $=' \&$ ' THEN PredCat $\leftarrow$ Internal

IF ConjForm=' and' THEN PredCat $\leftarrow$ External.

This very simple rule provides a baseline of $69.83 \%$.

\subsection{Classifiers}

The experiments were conducted using the WEKA toolkit [13]. This provides implementations of several machine learning algorithms, along with the data structures and code needed to perform data input and output, data filtering, and the evaluation and presentation of results.

After some initial exploration using a variety of algorithms for supervised machine learning available in WEKA, we chose the following: the Multilayer Perceptron (see [15]), two lazy algorithms (IBk and $\mathrm{K}^{*}$; see [16] and [17] respectively), and three tree algorithms: Random Tree (an algorithm for constructing a decision tree that considers $K$ random features at each node), Logistic Model Trees (see [18]) and J4.8 (see [19]). We also include here the results for Naïve Bayes and Sequential Minimal Optimization (see [20]), given the popularity of these methods in the field.

\section{Results}

\subsection{Evaluation Scheme}

For evaluation, we used the $k$-fold method with $k=10$, so that our data set of 600 examples was divided into ten folds by random selection of instances from the original data set. Then, for each of the folds, the classification models were built on the remaining 540 examples and tested on the held-out fold. The sum of correctly classified examples for all folds is the final result. There are some side effects of this evaluation approach, which we mention in Section7, however, it still makes more sense to use this approach for our small data set of 600 examples, than artificially dividing this set into even smaller training and test data sets. 
Table 4. Results for $k$-fold evaluation

\begin{tabular}{|l|rl|}
\hline Algorithm & Correctly classified (out of 600) \\
\hline \hline IBk & $84.00 \%$ & $(504)$ \\
\hline Random Tree & $83.83 \%$ & $(503)$ \\
\hline K $^{*}$ & $83.50 \%$ & $(501)$ \\
\hline SMO & $82.33 \%$ & $(494)$ \\
\hline Mult. Perc. & $82.17 \%$ & $(493)$ \\
\hline LMT & $81.17 \%$ & $(487)$ \\
\hline J4.8 & $79.50 \%$ & $(477)$ \\
\hline Naïve Bayes & $70.67 \%$ & $(424)$ \\
\hline Baseline & $69.83 \%$ & $(419)$ \\
\hline
\end{tabular}

Table 5. Detailed accuracy by category of a conjunction for results of IBk classifier

\begin{tabular}{|c|c|c|c|}
\hline Category & Precision & Recall & F-Measure \\
\hline \hline Name Internal & 0.814 & 0.876 & 0.844 \\
Name External & 0.872 & 0.897 & 0.885 \\
Right-Copy & 0.615 & 0.410 & 0.492 \\
Left-Copy & 0.800 & 0.462 & 0.585 \\
\hline weighted mean & 0.834 & 0.840 & 0.833 \\
\hline
\end{tabular}

Table 6. Confusion matrix for IBk

\begin{tabular}{|cccc|c|}
\hline Name Internal Name External Right Copy Left Copy & $\rightarrow$ classified as $\downarrow$ \\
\hline \hline 162 & 28 & 6 & 3 & Name Internal \\
18 & 314 & 17 & 11 & Name External \\
4 & 6 & 16 & 0 & Right Copy \\
1 & 2 & 0 & 12 & Left Copy \\
\hline
\end{tabular}

\subsection{Classification Results}

Table 4 presents the results achieved in the experiments. All algorithms scored above the baseline, though Naïve Bayes, with the worst result, was very close to the baseline.

The best classifier turned out to be IBk, the $K$-nearest neighbours algorithm. The precision, recall and F-measure for this case are presented in Table 5 Table 6 provides a confusion matrix with the desired and actual classification of examples. The best results are for Name Internal and Name External conjunctions. The low results for Right- and Left-Copy Separator conjunction types are mainly because of low recall for these categories: 0.410 and 0.462 , respectively. This is most likely caused by the fact that there are very few examples of these categories: $6.5 \%$ and $4.3 \%$, respectively (see Table 11).

We used the $\chi^{2}$ test for equality of distributions and a significance level of $90 \%$ to check whether the difference between the result of IBk and other algorithms is statistically significant; on this basis, we find that only the difference between the IBk algorithm and the Random Tree algorithm is no greater than chance.

It is interesting to note that the relatively simple Random Tree algorithm scored so highly. We tried different values for its parameter $K$, the number of randomly chosen attributes to be considered at each node. The result presented in the table is for $K=22$; for the default $K=1$, the algorithm correctly classified 490 examples.

\section{Analysis}

\subsection{Conjunction Category Indicators}

A statistical analysis of the data reveals some strong conjunction category indicators. 
For the Name External these are:

- a Month tag in the left conjunct (as in September and December);

- a Comp-Desig or Abbrev tag in the left conjunct (as in Alliance Technology Pty Ltd and Suco International or NLD and BRL Hardy); but there are exceptions: JP Morgan Investment Management Australia Ltd and Associates, Association of Mining \& Exploration Companies and ASX Settlement and Transfer Corporation, which are all Name Internal;

- a Month or PersDesig tag in the right hand conjunct (as in February and March or Mr R L Hanwright \& Mrs M J Hanwright; and

- a GivenName, Dir or Abbrev tag in the right hand conjunct, although there are exceptions: Beaches and Quay West Brisbane and SMDS and ATM WANS (both are of the Right-Copy Separator type).

The presence of a Son tag is a strong indicator of a Name Internal conjunction.

\subsection{Error Analysis}

We have demonstrated that with supervised machine learning over a simple set of features, we achieve a classification error rate of $16-18 \%$. We now provide some discussion of the classification errors made by the best-performing learner, the IBk algorithm.

InitCapped: Of the 96 misclassified examples, 38 (39.58\%) consist of a pattern consisting entirely of InitCapped tags. In such cases, classification ends up being determined on the basis of the ConjForm attribute: if the value is $\&$, then the conjunction is classified as being Name Internal, and if its value is and, the conjunction is classified as being Name External. Consequently, the following examples are misclassified: Victorian Casino and Gaming Authority, Coal Handling and Preparation Plan, Gas Supply and Demand Study, and Explanatory Memorandum \& Proxy Form.

At the same time, there were 96 InitCapped-only patterns that were classified correctly; this means that out of all 134 InitCapped-only patterns $71.64 \%$ were classified correctly, which is quite consistent with the previously-discussed baseline.

There were also another 11 misclassified instances consisting mainly of InitCapped tags along with some other tags; examples of these are: Australian Labor Party and Independent Members 〈Loc InitCapped Org and InitCapped InitCapped〉, Association of Mining \& Exploration Companies 〈CompDesig Of InitCapped \& InitCapped InitCapped〉 and Securities and Exchange Commission 〈InitCapped and InitCapped Org $\rangle$.

Long Patterns: Two misclassified instances were represented by relatively long patterns: for example, Fellow of the Australian Institute of Geoscientists and The Australasian Institute of Mining, represented by the 12-tag pattern 〈CompPos Of Det Loc Org Of InitCapped and Det Loc Org Of InitCapped $>$.

Other Interesting Cases: There were two cases of misclassified strings whose patterns themselves contained more common patterns as subsequences; in these cases, the information in the larger pattern was not insufficient to override the pull of the embedded pattern. One example is the string WD \& HO Wills Holdings Limited: being the name of a company, here the conjunction is Name-Internal, with the pattern / Initial Initial \& Initial Initial FamilyName CompDesig $\rangle$. However, this is incorrectly classified as 
containing a Right-Copy Separator conjunction, as is the case in the constituent pattern $\langle$ Initial Initial \& Initial Initial FamilyName .

The string Wayne Jones and Topsfield Pty Ltd, which in reality involves a Name External conjunction, was classified as Name Internal. We would note here that, in the absence of additional contextual information, conjunctions of person names and company names are often ambiguous even for humans.

Another related highly ambiguous type of example corresponds to the pattern $\langle$ FamilyName and FamilyName〉, which can either be a conjunction of two person names or just one company name.

We also note here the impact of the $k$-fold evaluation approach. Since a new model is built for each fold, it turns out that the IBk classifier assigned category Name Internal to instances of the pattern 〈InitCapped and InitCapped Org in one case, but assigned Right-Copy in another case. Consequently, both Federal and State Government (RightCopy), being in one fold, and Securities and Exchange Commission (Name Internal), being in another fold, were misclassified.

Other Observations: There are also some cases which we expected to be handled easily, but which turned out to be problematic. For example, D J Carmichael Pty Limited and Kirke Securities Ltd was classified as Name Internal, although it contains company designators in both conjuncts and the form of conjunction is and. Similarly, the string Department of Transport and Department of Main Roads (with the pattern OOrg Of InitCapped and Org Of InitCapped InitCapped, External $\rangle$ ) was classified as Name Internal.

Finally, there are around 15-20 examples for which it is difficult to provide a clear explanation for misclassification along the lines of the cases above; in these cases, the major issue is the classifier's ability to generalize the rules (which is not necessarily due to a deficiency in the algorithm, but perhaps due to the simple tagset we use).

\section{Conclusions and Future Work}

We have presented the problem of conjunction disambiguation in named entities and defined four categories of conjunction in candidate named entity strings. We defined the problem as one of classification and showed that it can be handled well using supervised machine learning algorithms and a limited set of name-internal features.

Given the similarity in results for most of the different machine-learned classifiers we used, we conclude that a significant improvement of results lies in a richer feature selection rather than in choice of the classifier. This conclusion is also supported by the fact that some examples are difficult for a human to classify without wider context or domain knowledge.

A number of issues arise in the work reported here as candidates for future work. We have restricted ourselves to candidate strings which contain a single conjunction; however, there are of course cases where multiple conjunctions appear. One category consists of examples like Audited Balance Sheet and Profit and Loss Account, where again the kinds of syntactic ambiguity involved would suggest a more syntacticallydriven approach would be worth consideration. Another category consists of candidate named entity strings that contain commas as well as lexicalised conjunctions. 
A rudimentary analysis of frequently occurring $n$-grams in our corpus makes it clear that some strings containing conjunctions appear frequently. For example, in our corpus there are 296 occurrences of the string Quarter Activities and Cashflow Report? making it the most frequent 5 -gram. Moreover, there are another 34 occurrences of this string with the conjunction $\&$ in place of and, and another six strings with the variant spelling Cash Flow. In any real application context, it would make sense to filter out these common cases via table lookup before applying a machine learning process to classify the remaining conjunctions. This kind of preprocessing could identify frequent strings containing either Name Internal or Name External conjunctions. Another form of preprocessing could involve the analysis of abbreviations: for example, in the string ASX Settlement and Transfer Corporation (ASTC), the abbreviation ASTC could be used to decide that the preceding conjunction has the category Name Internal.

More generally, there are three directions in which we might move in order to further improve performance.

First, we can always use larger gazetteers to reduce the number of tokens that can only be tagged as InitCapped. This, of course, has a cost consequence; in current work, we are exploring how performance on this task improves as larger numbers of frequent name elements from the corpus are incorporated into the gazetteers. Another consequence of extending gazetteers is the problem of the same token being in two or more gazetteers, for example Location and FamilyName. A naive approach would be to assign these tokens the catch-all InitCapped tag, but since this is what we want to avoid, we could also assign all the ambiguous tags and indicate this fact in the feature vector. This would require a redesign of the feature vector.

Second, we can make more sophisticated use of the name internal properties of the candidate string. This includes, as noted above with regard to the Exchanges example, taking account of the syntactic number of the constituent tokens. Armed with a part of speech tagger, we could also attempt heuristic chunking of the candidate strings which might assist in determining conjunction type; and a resource like WordNet might be used to identify terms with shared superordinates, as in the Paper and Boards example.

Third, we can extend the learning process to take account of contextual features. As noted earlier, there are cases where the local context cannot be easily determined, but in many cases local syntactic information such as the number of an associated verb can serve to distinguish the type of conjunction being used. However, as demonstrated here, it is already possible to achieve a high level of accuracy without recourse to name external features; as we noted earlier, this is important in our domain, where names often appear in tables, making local context unavailable.

\section{Acknowledgements}

We acknowledge the support of the Capital Markets Cooperative Research Centre in carrying out this work. The work was carried out while the second author was a visiting scholar at Macquarie University. The final version of this paper was prepared while the second author was a guest researcher at DFKI, Saarbrücken.

\footnotetext{
${ }^{2}$ This appears frequently as a substring of longer expressions like First Quarter Activities and Cashflow Report, Second Quarter Activities and Cashflow Report, and so on.
} 


\section{References}

1. Grishman, R., Sundheim, B.: Design of the MUC-6 Evaluation. In: Sixth Message Understanding Conference (MUC-6): Proceedings of a Conference held in Columbia, Maryland, November 6-8, 1995, Los Altos, Ca., Morgan Kaufmann (1995)

2. Grishman, R., Sundheim, B.: Message Understanding Conference-6: A Brief History. In: COLING 1996 Volume 1: The 16th International Conference on Computational Linguistics, Los Altos, Ca., Morgan Kaufmann (1996)

3. Sang, E.F.T.K.: Introduction to the CoNLL-2002 Shared Task: Language-Independent Named Entity Recognition. In Roth, D., van den Bosch, A., eds.: Proceedings of the 6th Conference on Natural Language Learning, Taipei, Taiwan (2002) 155-158

4. Sang, E.F.T.K., Meulder, F.D.: Introduction to the CoNLL-2003 Shared Task: LanguageIndependent Named Entity Recognition. In Daelemans, W., Osborne, M., eds.: Proceedings of the 7th Conference on Natural Language Learning, Edmonton, Canada (2003) 142-147

5. Rau, L.F.: Extracting company names from text. In: Proceedings of the Seventh Conference on Artificial Intelligence Applications, IEEE (1991) 189-194

6. Coates-Stephens, S.: The analysis and acquisition of proper names for the understanding of free text. Computers and the Humanities V26 (1992) 441-456

7. McDonald, D.D.: Internal and external evidence in the identification and semantic categorization of proper names. In: B. Boguraev and J. Pustejovsky, editors, Corpus processing for lexical acquisition, pages 21-39. (1996)

8. Mikheev, A., Grover, C., Moens, M.: Description of the LTG System Used for MUC-7. In: Proc. of MUC-7 Conf. (1998)

9. McDonald, R., Crammer, K., Pereira, F.: Flexible text segmentation with structured multilabel classification. EMNLP (2005)

10. Solorio, T.: Improvement of Named Entity Tagging by Machine Learning. Technical Report CCC-04-004, Coordinacin de Ciencias Computacionales (2004)

11. Steedman, M.: Dependency and Coordination in the Grammar of Dutch and English. Language 61 (1985) 523-568

12. Dale, R., Calvo, R., Tilbrook, M.: Key Element Summarisation: Extracting Information from Company Announcements. In: Proc. of the 17th Australian Joint Conf. on AI, 7th-10th Dec. 2004, Australia. (2004)

13. Witten, I.H., Frank, E.: Data Mining: Practical machine learning tools and techniques. Morgan Kaufmann, San Francisco (2005)

14. Holte, R.C.: Very simple classification rules perform well on most commonly used datasets. Machine Learning 11 (1993) 63-91

15. Rojas, R.: Neural networks: a systematic introduction. Springer-Verlag New York, Inc., New York, NY, USA (1996)

16. Aha, D.W., Kibler, D., Albert, M.K.: Instance-based learning algorithms. Mach. Learn. 6 (1991) 37-66

17. Cleary, J.G., Trigg, L.E.: $\mathrm{K}^{*}$ : An Instance-based Learner Using an Entropic Distance Measure. In: Proceedings of the 12th International Conference on Machine Learning, Morgan Kaufmann (1995) 108-114

18. Landwehr, N., Hall, M., Frank, E.: Logistic Model Trees. Machine Learning 59(1/2) (2005) 161-205

19. Quinlan, J.R.: C4.5: programs for machine learning. Morgan Kaufmann Publishers Inc., San Francisco, CA, USA (1993)

20. Platt, J.C.: Fast training of support vector machines using sequential minimal optimization. In: Advances in Kernel Methods: Support Vector Learning, Cambridge, MA, USA, MIT Press (1999) 185-208 